\title{
A CONDITION FOR ANALYTIC STRUCTURE ${ }^{1}$
}

\author{
RICHARD F. BASENER
}

\begin{abstract}
Let $X$ be a compact Hausdorff space, $A$ a uniform algebra on $X, M$ the maximal ideal space of $A$. Let $f \in A$ and let $W$ be a component of $C \backslash f(X)$. Suppose that, for all $\lambda \in W, f^{-1}(\lambda)=$ $\{x \in M \mid f(x)=\lambda$. $\}$ is at most countable. Then there is an open dense subset $U$ of $f^{-1}(W)$ which can be given the structure of a onedimensional complex analytic manifold so that for all $g \in A, g$ is analytic on $U$.
\end{abstract}

Let $X$ be a compact Hausdorff space, let $A$ be a uniform algebra on $X$, and let $M$ be the maximal ideal space of $A$.

THEOREM. Let $f \in A$ and let $W$ be a component of $C \backslash f(X)$. Suppose that for all $\lambda \in W, f^{-1}(\lambda)=\{x \in M \mid f(x)=\lambda\}$ is at most countable. Then there is an open dense subset of $f^{-1}(W)$ which can be given the structure of a onedimensional complex analytic manifold so that the functions in $A$ become analytic there.

This theorem partially generalizes results for the case when $f^{-1}(\lambda)$ is finite for all $\lambda$ in a sufficiently large subset of $W$, which are essentially contained in a paper of E. Bishop ([1], see Theorem 11.2 in [2]). A key result in the proof of this earlier result is also important in the proof of our theorem (see Lemma 13 in [1] or Theorem 10.7 in [2]).

Definition. Let $p \in M$, and let $\Phi$ be a continuous one-to-one map from $\{|z|<1\}$ into $M$, with $\Phi(0)=p$. The set $\{\Phi(z)|| z \mid<1\}$ is called an analytic disk through $p$ if for all $h \in A, h \circ \Phi$ is analytic on $\{|z|<1\}$.

LEMMA 1. Let $f \in A$ and suppose that:

(a) $|f|=1$ on $X$;

(b) $\exists p \in M$ with $f(p)=0$;

(c) $\exists$ a closed subset $\Gamma_{0}$ of $\{|z|=1\}$ having positive linear measure such that for each $\lambda \in \Gamma_{0}$ there is a unique point $q \in X$ with $f(q)=\lambda$.

Then $f^{-1}(\{|z|<1\})$ is an analytic disk through $p$.

Presented to the Society, October 13, 1971; received by the editors December 30, 1971.

AMS 1970 subject classifications. Primary 46J10, 46J15.

Key words and phrases. Analyticity, maximal ideal space.

1 This research was supported in part by the National Science Foundation under grant GP-28574.

(c) American Mathematical Society 1972 
We also need the following elementary result (Lemma 11.1 in [2]).

LeMma 2. Let $f \in A$ and let $W$ be a component of $C \backslash f(X)$. Fix $\lambda \in W$. If $f$ takes on the value $\lambda$ on $M$, then $f$ takes on every value in $W$ on $M$.

One further result is needed.

Lemma 3. Let $f \in A$ and let $W$ be a component of $C \backslash f(X)$. Let $z \in W$, and suppose that $f^{-1}(z)$ is at most countable. Given a neighborhood $\mathcal{O}$ of a point $x \in f^{-1}(z)$, there is a compact neighborhood $N$ of $x$ with the properties that:

(a) $N$ is A-convex, i.e., the maximal ideal space of $\left.\bar{A}\right|_{N}$ is $N$;

(b) $z \notin f(\partial N)$;

(c) $N \subseteq \mathcal{O} \cap f^{-1}(W)$.

Furthermore, $f(N)$ is a neighborhood of $z$ in $C$.

Proof of Lemma 3. Choose $\varepsilon>0$ and functions $g_{1}, g_{2}, \cdots, g_{n} \in A$ so that

$$
N=\left\{y \in M|| g_{j}(y)-g_{j}(x) \mid \leqq \varepsilon, 1 \leqq j \leqq n\right\} \subseteq f^{-1}(W) \cap \mathcal{O} .
$$

Since $f^{-1}(z)$ is at most countable, we may adjust $\varepsilon$ so that, for all $y \in f^{-1}(z)$, $\left|g_{j}(y)-g_{j}(x)\right| \neq \varepsilon, j=1,2, \cdots, n$. Now if $y \in \partial N$, then $\left|g_{j}(y)-g_{j}(x)\right|=\varepsilon$ for some $j$, so $f^{-1}(z) \cap \partial N=\varnothing$ and it is clear that (a), (b) and (c) are satisfied.

By the local maximum modulus principle, the Shilov boundary of $\left.\bar{A}\right|_{N}$ is contained in $\partial N$ (note that $N$ does not meet the Shilov boundary of $A$ ). Hence $\left.\bar{A}\right|_{N}$ may be regarded as a uniform algebra on $\partial N$. Also, $z \in$ $f(N) \backslash f(\partial N)$. By Lemma 2, $f(N)$ contains the component of $z$ in $C \backslash f(\partial N)$, so $f(N)$ is a neighborhood of $z$ as claimed.

ProOf OF THEOREM. We will prove the following.

Assertion. If $f^{-1}(W)$ is nonempty, $\exists p \in f^{-1}(W)$ with a neighborhood in $M$ which is an analytic disk through $p$.

Assume for the moment that the assertion has been established. Given any $x \in f^{-1}(W)$ and any neighborhood $\mathcal{O}$ of $x$, we may apply Lemma 3 to find a compact neighborhood $N$ of $x$ with properties (a), (b) and (c). Then the assertion may be applied to $\left.\left.f\right|_{N} \in \bar{A}\right|_{N}$, to yield a point $p \in \mathcal{O}$ with a neighborhood which is an analytic disk through $p$. Thus the set of such points is dense in $f^{-1}(W)$, and it is obviously open, so the Theorem follows.

ProOf OF ASSERTION. Suppose, on the contrary, that no point of $f^{-1}(W)$ has a neighborhood which is an analytic disk. We will use this assumption to find a point $z \in W$ such that $f^{-1}(z)$ is uncountable, contradicting the hypothesis of the Theorem. The uncountable set will consist of limit points obtained from sequences $\left\{x_{i_{1} i_{2} \cdots i_{n}}\right\}_{n=1}^{\infty}$ in $M$, which we will now define inductively. 
Step 1. Since $f^{-1}(W)$ is nonempty, by Lemma 2 we have $f\left(f^{-1}(W)\right)=W$, so by Lemma 1 and the assumption that no point of $f^{-1}(W)$ has a neighborhood which is an analytic disk, there are distinct points $x_{0}, x_{1} \in M$ and a corresponding $z_{1} \in W$ such that $f\left(x_{0}\right)=f\left(x_{1}\right)=z_{1}$. By Lemma 3 there are disjoint compact $A$-convex neighborhoods $N_{i_{1}}$ of $x_{i_{1}}$ with the properties $N_{i_{1}} \subseteq f^{-1}(W), z_{1} \notin f\left(\partial N_{i_{1}}\right)$, and $f\left(N_{i_{1}}\right)$ is a neighborhood of $z_{1}, i_{1}=0,1$. Choose $\varepsilon_{1}$ with $0<\varepsilon_{1}<1$ so that

$$
\left\{\left|z-z_{1}\right| \leqq \varepsilon_{1}\right\} \subseteq f\left(N_{i_{1}}\right) \backslash f\left(\partial N_{i_{1}}\right), \quad i_{1}=0,1 .
$$

Fix $i_{1}$ and look at

$$
M_{i_{1}}=\left\{y \in N_{i_{1}}|| f(y)-z_{1} \mid \leqq \varepsilon_{1}\right\}=N_{i_{1}} \cap f^{-1}\left(\left\{\left|z-z_{1}\right| \leqq \varepsilon_{1}\right\}\right) .
$$

$M_{i_{1}}$ is a nonempty compact $A$-convex set, so the maximal ideal space of $\left.\bar{A}\right|_{M_{i_{1}}}$ is $M_{i_{1}}$. Let $X_{i_{1}}=\left\{y \in N_{i_{1}}|| f(y)-z_{1} \mid=\varepsilon_{1}\right\}$.

Observe that $\partial M_{i_{1}} \subseteq\left(\partial N_{i_{1}} \cup X_{i_{1}}\right) \cap M_{i_{1}}=X_{i_{1}}$ since $\partial N_{i_{1}} \cap M_{i_{1}}=\varnothing$ by $\left(\mathrm{A}_{1}\right)$. Also observe that $M_{i_{1}}$ does not meet the Shilov boundary of $A$. By the local maximum modulus principle, the Shilov boundary of $\left.\bar{A}\right|_{M_{i_{1}}}$ is contained in $X_{i_{1}}$. Thus we may apply Lemma 1 with $X=X_{i_{1}}, A=\left.\bar{A}\right|_{X_{i_{1}}}$, $M=M_{i_{1}}, f=\left(f-z_{1}\right) / \varepsilon_{1}$, to conclude that (since there is no analytic disk through any point of $\left.f^{-1}(W)\right)$ :

$$
\begin{aligned}
\text { linear measure }\left\{z \in C|| z-z_{1} \mid=\right. & \varepsilon_{1} \\
& \text { and } \left.f^{-1}(z) \text { is unique in } N_{i_{1}}\right\}=0 .
\end{aligned}
$$

(Note that $f^{-1}(z) \cap N_{i_{1}}=f^{-1}(z) \cap M_{i_{1}}$ if $\left|z-z_{1}\right|=\varepsilon_{1}$. The set described in statement $\left(B_{1}\right)$ is measurable since Lemma 3 implies that it is closed. In fact, suppose that $\zeta_{1}, \zeta_{2}, \cdots \in C, \zeta_{j} \rightarrow \zeta \in C,\left|\zeta_{j}-z_{1}\right|=\varepsilon_{1}$ and there is precisely one $\alpha_{j} \in N_{i_{1}}$ such that $f\left(\alpha_{j}\right)=\zeta_{j}, j=1,2, \cdots$. Let $\alpha \in f^{-1}(\zeta) \cap N_{i_{1}}$ and let $V$ be any neighborhood of $\alpha$ in $M$. By Lemma 3, $f\left(V \cap N_{i_{1}}\right)$ is a neighborhood of $\zeta$ in $C$, hence $\zeta_{j}$ is in $f\left(V \cap N_{i_{1}}\right)$ for all $j \geqq j_{0}$ for some $j_{0}$. But $f^{-1}\left(\zeta_{j}\right) \cap N_{i_{1}}=\left\{\alpha_{j}\right\}$, so $\alpha_{j}$ is in $V$ for $j \geqq j_{0}$. Thus $\alpha_{j}$ converges to $\alpha$, and $\alpha$ is unique.)

This completes the first step.

Inductive hypothesis. Suppose that $z_{n} \in W, x_{a} \in M, N_{a} \subseteq M$ (where $\left.a=i_{1} \cdots i_{n}\right)$ and $\varepsilon_{n}>0$ have been chosen for $i_{j}=0,1,1 \leqq j \leqq n$. Assume that they satisfy the conditions: the $2^{n}$ points $x_{a}$ are all distinct; $f\left(x_{a}\right)=z_{n}$; $N_{a}$ is a compact $A$-convex neighborhood of $x_{a} ; N_{a} \cap N_{b}=\varnothing$ (where $b=$ $\left.j_{1} \cdots j_{n}\right)$ unless $i_{1}=j_{1}, \cdots, i_{n}=j_{n} ; N_{a} \subseteq$ interior $N_{i_{1} \cdots i_{n-1}}$, if $n>1 ; \varepsilon_{n}<1 / n^{2}$;
$\left(\mathrm{A}_{n}\right)$
$\left\{\left|z-z_{n}\right| \leqq \varepsilon_{n}\right\} \subseteq f\left(N_{a}\right) \backslash f\left(\partial N_{a}\right) ;$
linear measure $\left\{z \in C|| z-z_{n} \mid=\varepsilon_{n}\right.$

$$
\text { and } \left.f^{-1}(z) \text { is unique in } N_{a}\right\}=0 \text {. }
$$


Step $n+1$. By $\left(\mathrm{B}_{n}\right), \exists z_{n+1} \in\left\{\left|z-z_{n}\right|=\varepsilon_{n}\right\}$ such that for all $i_{1} \cdots i_{n}$, there are two distinct points $x_{c} \in N_{a}$ (where $c=i_{1} \cdots i_{n+1}, i_{n+1}=0,1$ ) with $f\left(x_{c}\right)=z_{n+1}$. By $\left(\mathrm{A}_{n}\right)$, we have $x_{c} \in N_{a} \backslash f^{-1}\left(f\left(\partial N_{a}\right)\right)$. Fix $a=i_{1} \cdots i_{n}$ and choose disjoint open sets $\mathcal{O}_{0}, \mathcal{O}_{1}$ with

$$
x_{c} \in \mathcal{O}_{i_{n+1}} \subseteq N_{a} \backslash f^{-1}\left(f\left(\partial N_{a}\right)\right), \quad i_{n+1}=0,1 .
$$

Fix $i_{n+1}=0$ or 1 and apply Lemma 3 with

$$
A=\left.\bar{A}\right|_{\partial N_{a}}, \quad X=\partial N_{a}, \quad M=N_{a}, \quad f=\left.f\right|_{N_{a}},
$$

$W=$ component of $z_{n+1}$ in $C \backslash f\left(\partial N_{a}\right), \quad z=z_{n+1}, \quad \mathcal{O}=\mathcal{O}_{i_{n+1}}, \quad x=x_{c}$.

We conclude that there exist disjoint compact $A$-convex neighborhoods $N_{c}$ of $x_{c}, i_{n+1}=0,1$, with the properties $z_{n+1} \notin f\left(\partial N_{c}\right)$,

$$
N_{c} \subseteq N_{a} \backslash f^{-1}\left(f\left(\partial N_{a}\right)\right),
$$

$f\left(N_{c}\right)$ is a neighborhood of $z_{n+1}$. Do this for all indices $c=i_{1} \cdots i_{n+1}$, $i_{j}=0,1,1 \leqq j \leqq n+1$.

From the definition of the $N_{c}$ it follows that we can choose an $\varepsilon_{n+1}$ with $0<\varepsilon_{n+1}<1 /(n+1)^{2}$ so that for all $i_{1} \cdots i_{n+1}$ we have

$$
\left(\mathrm{A}_{n+1}\right) \quad \quad\left\{\left|z-z_{n+1}\right| \leqq \varepsilon_{n+1}\right\} \subseteq f\left(N_{c}\right) \backslash f\left(\partial N_{c}\right) .
$$

Fix $c=i_{1} \cdots i_{n+1}$ and define

$$
\begin{aligned}
M_{c} & =\left\{y \in N_{c}|| f(y)-z_{n+1} \mid \leqq \varepsilon_{n+1}\right\}, \\
X_{c} & =\left\{y \in N_{c}|| f(y)-z_{n+1} \mid=\varepsilon_{n+1}\right\} .
\end{aligned}
$$

As in Step 1, the maximal ideal space $\left.\bar{A}\right|_{M_{c}}$ is $M_{c}$ and the Shilov boundary of $\left.\bar{A}\right|_{M_{c}}$ is contained in $X_{c}$. $\left(\left(\mathrm{A}_{n+1}\right)\right.$ guarantees that $\partial M_{c} \subseteq X_{c}$.) We may therefore apply Lemma 1 with

$$
X=X_{c}, \quad A=\left.\bar{A}\right|_{\Upsilon_{c}}, \quad M=M_{c}, \quad f=\left(f-z_{n+1}\right) / \varepsilon_{n+1} .
$$

We conclude that

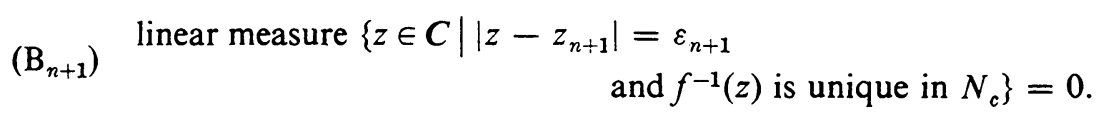

This equation holds for all indices $c=i_{1} \cdots i_{n+1}, i_{j}=0,1,1 \leqq j \leqq n+1$, and the induction is complete.

By the above construction $\left\{z_{n}\right\}$ is Cauchy, so $z_{n} \rightarrow z$ for some $z \in C$; $z \in W$ since, for each $n, z_{n} \in f\left(N_{a}\right) \subseteq f\left(N_{0}\right) \cup f\left(N_{1}\right)$, a compact subset of $W$.

Let $I=\left(i_{1}, i_{2}, \cdots, i_{n}, \cdots\right)$ be an infinite sequence of 0 's and 1 's. Some subnet of the sequence $x_{i_{1}}, x_{i_{1} i_{2}}, x_{i_{1} i_{2} i_{3}}, \cdots$ converges to a point $x_{I} \in M$, and by continuity $f\left(x_{I}\right)=z$. In this way we associate an $x_{I}$ with each $I$. 
Since there are uncountably many distinct $I$ 's, the proof will be complete if we show that $x_{I} \neq x_{J}$ whenever $I \neq J$. Suppose therefore that $i_{1}=j_{1}$, $i_{2}=j_{2}, \cdots, i_{m}=j_{m}, i_{m+1} \neq j_{m+1}$. We know that $x_{i_{1}} \in N_{i_{1}}, x_{i_{1} i_{2}} \in N_{i_{1} i_{2}}, \cdots$ and $N_{i_{1}} \supseteq N_{i_{1} i_{2}} \supseteq N_{i_{1} i_{2} i_{3}} \cdots$ by the inductive hypotheses. Thus $x_{I} \in \bigcap_{n=1}^{\infty} N_{a}$. Similarly $x_{J} \in \bigcap_{n=1}^{\infty} N_{b}$ where $b=j_{1} \cdots j_{n}$. But $N_{i_{1} \cdots i_{m+1}} \cap N_{j_{1} \cdots j_{m+1}}=\varnothing$ since $i_{m+1} \neq j_{m+1}$, so $x_{I} \neq x_{J}$.

B. Cole has pointed out that if one merely assumes that $f^{-1}(\lambda)$ is countable for $\lambda$ in a subset of $W$ of positive plane measure, the same proof shows that there is at least one point $p \in f^{-1}(W)$ with a neighborhood in $M$ which is an analytic disk through $p$.

I would like to thank J. Wermer, who pointed out to me the question answered by the Theorem, for many helpful discussions and suggestions.

\section{REFERENCES}

1. E. Bishop, Holomorphic completions, analytic continuation, and the interpolation of semi-norms, Ann. of Math. (2) 78 (1963), 468-500. MR 27 \#4958.

2. J. Wermer, Banach algebras and several complex variables, Markham, Chicago, Ill., 1971.

Department of Mathematics, Brown University, Providence, Rhode Island 02912

Current address: Department of Mathematics, Yale University, New Haven, Connecticut 06520 